\title{
Design and Implementation of Parking Monitoring System of Bike Sharing In the Electronic Barrier
}

\author{
Wei SHEN ${ }^{1, a}$ and Jiao $\mathrm{LI}^{2, \mathrm{~b}}$ \\ ${ }^{1}$ School of Computer Science and Engineering,Wuhan Institute of Technology,Wuhan,Hubei,China \\ ${ }^{2}$ PowerChina Hubei Electric Engineering Corporation,Wuhan,Hubei,China \\ ${ }^{a}$ wws.wit@163.com, b13971027621@139.com
}

Keywords: Electric Barrier, GPS Auto Monitor, Bike Sharing, Map Matching.

\begin{abstract}
Electric barrier, which is one of the most vital function in GPS auto monitor system demands, has being widely applied in many fields liking bike sharing. This paper presents an intelligent GPS auto monitor system and describes the design and implement of electric barrier on a Shared bike. By means of map matching method and GPS to realize the parking of the Shared cycle in the designated area, it is more effective to use the sharing cycle to facilitate the travel of the mass of people. On the vehicle monitoring map, draw polygons of the electronic fence, when the vehicle in the area to meet the appropriate conditions, can alarm or do some other linkage processing. By using the GPS coordinate of the vehicle and the map information of the electronic fence, the mathematical model is built, and the algorithm is designed. This software is implemented by programming.
\end{abstract}

\section{Introduce}

The sharing cycle refers to the bicycle sharing service provided by enterprises in campus, subway station, bus station, residential area, commercial district, public service area and is a time-sharing rental mode. Bike Sharing is a new kind of sharing economy. With the emergence of various sharing bikes in the market, people travel in a very convenient way. The popularity of this method has greatly improved people's time of appearance. Especially in some big cities, with the increasing number of subway, the urbanization of village is becoming more and more choose shared cycling, connected to the community and, estuary metro subway travel got greatly alleviate the problem of the last kilometer. Shared cycling is the essence of which is a new type of vehicle rental business - bike rental business, mainly depend on the carrier for the bike (bicycle), can make full use of the city because of the rapid economic development of bicycle travel low status, to maximize the use of public roads pass rate, at the same time has the effect of healthy body. But the problem of that followed was the management of bicycles, especially parking problems. How to maximize the use of shared bikes, parking problem is a very worthwhile problem. Here, we can use the electronic fence to realize the parking monitoring of bicycles.

Problems existing in the development of this article from the Shared a bicycle, how to standardized management, raises the electronic fence this technology, Shared cycling was introduced in detail, the electronic fence, electronic fence said, storage, map matching and position determination and automatic alarm, and other aspects, and finally introduces the electronic fence in Shared bicycle parking monitoring system in the implementation process.

\section{Polygon region processing.}

As shown in fig. 1 the judge point $\mathrm{p}$ in the polygon method is: use the level of the point $\mathrm{p}$ to coordinate and polygon intersection, get a number of intersection points, if the number of intersection point on either side of the point $\mathrm{p}$ is an odd number when, that point $\mathrm{p}$ within the polygon, namely the plumb line interior-point method. This method is suitable for arbitrary polygons, including convex polygons and concave polygons, and is suitable for polygons with holes. 
When processing data in the database, can will first polygon circumscribed rectangle as district, choose meet rectangle points, after can be in to determine whether a selected point in polygon area, so that you can to a certain extent to improve the performance of database operations[1].

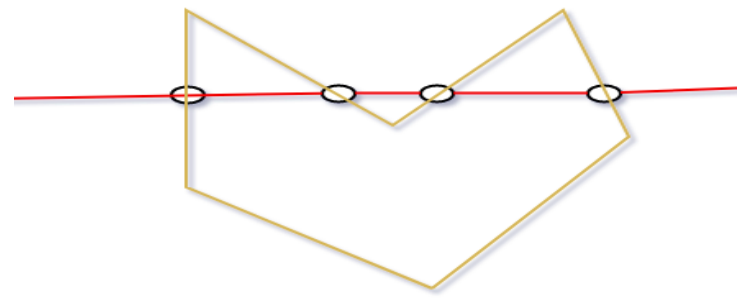

Figure 1. For Polygon region processing.

\section{Presentation and Storage of Electronic Fence}

Vehicles and electronic fence is a many-to-many relationship $(\mathrm{N}, \mathrm{N})$, which shows an car associates multiple electronic fence, and an electronic fence is also associated with multiple vehicles, so saved in the database, in addition to vehicles and electronic fence of the basic properties, but also saved both direct mapping relationship, the following table structure to store the mapping relationship (Table I). In the terminal, the relevant data is uploaded to the terminal Flash by the GPRS network for the real-time interpretation of the vehicle.

Table I. Shared cycle - electronic fence mapping table

\begin{tabular}{|c|c|}
\hline Field & Type \\
\hline Bicycle_id(primary key) & unsignen int \\
\hline Electric_Barner_id & unsignen int \\
\hline
\end{tabular}

\section{Map Matching}

Map Matching Algorithm (Map Matching, MM) is a high precision of navigation electronic maps to vehicle positioning error modification of a software method which can accurately match the vehicle to the actual road. Its principle is to pass positioning module for the position of the vehicle information, and then compare to and match the road network information, ultimately determine the cars in the road network, road, and solve the vehicle position of real time of current, at last can be visually displayed on the constructed map in real time[2].

The basic idea of map matching algorithm is through the GPS track and vector map of the target of the link object matching, looking for targets of the current traffic roads or channel, and will target the current GPS anchor point projection onto the road or waterway. When GPS coordinate values of output off the road maps or channel chain, monitoring platform based on a certain algorithm finds the nearest way or channel chain and the target accurately displayed in its position in the road or waterway. Looking for targets currently driving road is the key to the problem, the basic idea is to GPS target track of adjacent area search all route combinations, the combination of route was observed respectively with GPS track matching measures, in order to best match the combination of the measurement line as the target current driving route. Therefore, the map matching algorithm requires that digital electronic maps have higher accuracy in order to provide higher positioning accuracy[3,4].

This can be seen below shown as fig.2.First of all, based on GPS positioning data extraction, data fusion technology such as coordinate transformation and storage, the position of the target vehicle, and then based on the current map matching algorithm to find the location of the vehicle, and finally by the remote server stored in the database, so as to accurately on a smartphone shows the locationtarget vehicle[1]. 


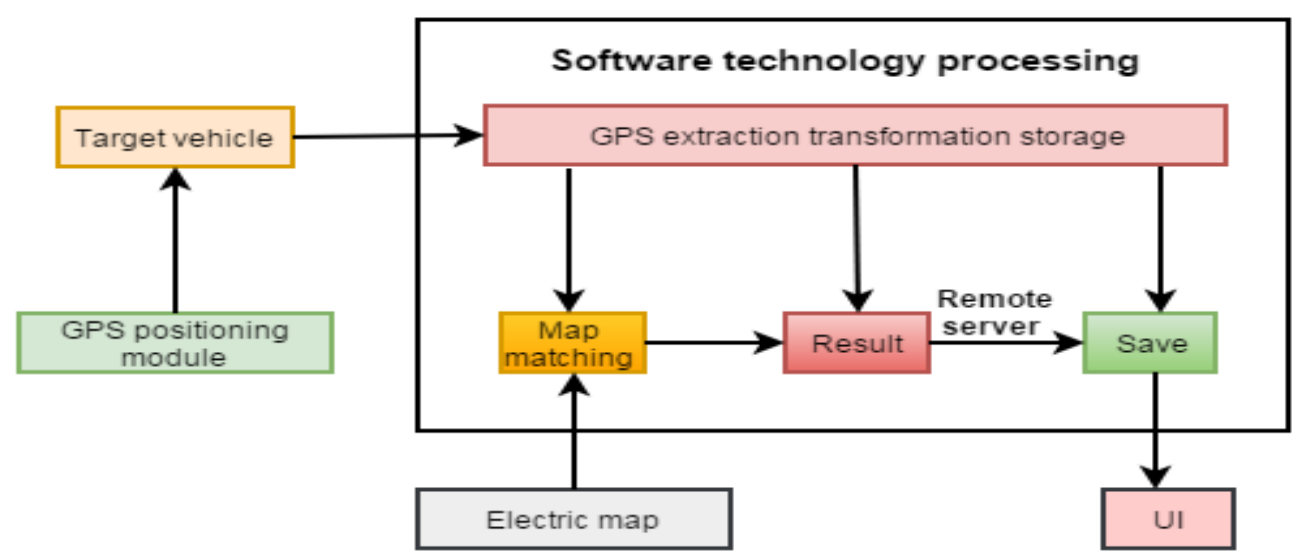

Figure 2. The process of map matching design

\section{Parking Position Determination}

Determine the core idea is Shared bicycle parking location through GPS coordinates of the system output is in the choice of green area, if it is, you can stop pay cost, otherwise will be parked near the green area on the map[5].

This paper describes the cross boundary decision algorithm of polygon.

Definition:

P: point position of any vehicle;

$\mathrm{V}$ : polygon area is adjacent to two points up end to end a collection of line segments, including the last segment starting point is the last point, the end is the first point, constitute a closed polygon.

$\mathrm{V}=\{\mathrm{Li}\}, \mathrm{i}=3 \ldots \mathrm{N}, / /$ where $\mathrm{Li}$ means a line segment.

$\mathrm{C}$ : the number of lines at which the boundary line of $\mathrm{V}$ is intersected with the base line of $\mathrm{P}$.

$\mathrm{C}=\{\mathrm{Ci}\}, \mathrm{i}=0 \ldots \mathrm{M}, 0<=\mathrm{M}<\mathrm{N}, \mathrm{Ci}$ is the intersection of line $\mathrm{I}$ segment and $\mathrm{P}$ reference line.

$\mathrm{Ci}=\mathrm{f}(\mathrm{P}, \mathrm{Li}) ; \mathrm{F}$ : the determination function of line segment intersection, and $\mathrm{Ci}=\{0,1\}$.

So, whether it crosses the line $\mathrm{B}: \mathrm{B}=\mathrm{C} \% 2$.

Algorithm complexity analysis: for a polygonal region, only need to traverse the boundary line once, so the complexity is $\mathrm{O}(\mathrm{n})$. Here are the specific algorithms.

Typedef struct// define the coordinate structure

\{DWORD x;//longitude

DWORD y;//latitude \}COORDINATE

BOOL JudgelnBarrier(const COORDINATE pos, const COORDINATEfl\&barrier)

\{barrier: // Electronic fence. There is a set of points spliced together

pos: // Determine the location of the vehicle

int $\mathrm{nCross} \mathrm{Cnt}=\mathrm{O}: / /$ The number of times crossing an electronic fence

int nCompareTimes $=\mathrm{O}$ :

int nSize=sizeof(barrier)/sizeof(COORDINATE);

B00L blnBarrier=false;

COORDINATE *Bext;

next=\&barrier[0];

BOOL bFront $=($ next $->y-p o s . y>0)$ ?true: fase;

next++:

// through polygonal region. Calculate the number of points of intersection

while(nCompareTimes $<$ nSize)

$\{$ BOOL bNext $=($ next- $>y-$ pos. $y>0)$ ? true: false;

if $(\mathrm{bNext}==\sim \mathrm{bFront})$;

nCrossCnt++// It intersects. Cumulative intersecting 
bFront=bNext; // The next endpoint becomes the current endpoint if (nCompareTimes $==$ nSize-1) // To the last line segment of the electronic fence next=\&barrier[0]; // The starting point becomes the endpoint of the last line else next++ // The starting point becomes the endpoint of the last line \} // through the polygon region, the number of intersecting points is odd, in the region; Otherwise, outside the area.

return $((\mathrm{nCrossCnt} \% 2 !=0)$ ?true: false $) ; \quad\}$

\section{Alert Processing}

After the electronic fence is set up, download through GPRS networks to be monitored in the mobile phone in a flash, parking during vehicle running, the mobile phone accepts a GPS point, and MCU performs a vehicle current position compared with the electronic fence area, if judged to be fall into electronic fence area, can stop pay cost, complete the automatic lock the car, otherwise, cannot complete stop billing, need a real-time map by a mobile phone to stop pay cost, by the electronic fence near the green area complete lock the car process.

\section{Conclusion}

This paper introduces the advantages of sharing bicycles, and focuses on the acquisition, representation, storage, matching, judgment and automatic alarm of electronic fence. Electronic fence is analysed in detail on the design and the realization of the automatic alarm in the Shared cycling process, realize the sharing of bicycle to park in the process of parking specification, and is more convenient to travel problems of most people.

A good effect was achieved in the practical application, and the results shown in Fig.3,4 and 5 showed the effect of the vehicle on the map immediately after entering the rectangle, circle and polygon area.If combined with other conditions out of the area into the area, such as vehicle speed in and out of time, area, and area number, and other conditions, is able to design and implement of more complicated procedures, in order to meet more applications.With the progress of science and technology, the improvement of the legal system, as well as the improvement of quality of people, believe that soon, Shared cycling does real convenience, not a nuisance to do green travel of an important means of transportation.

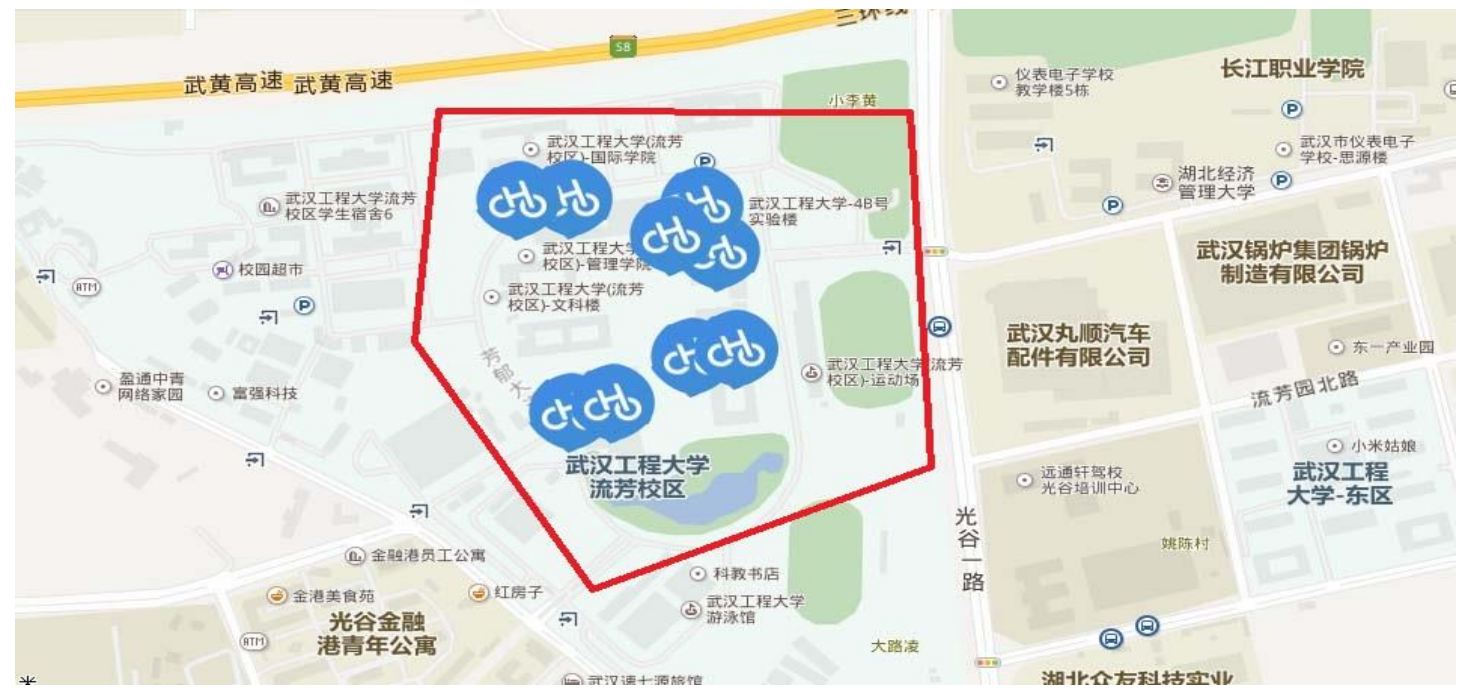

Figure 3 . The effect of the vehicle on the map immediately after entering the polygon area. 


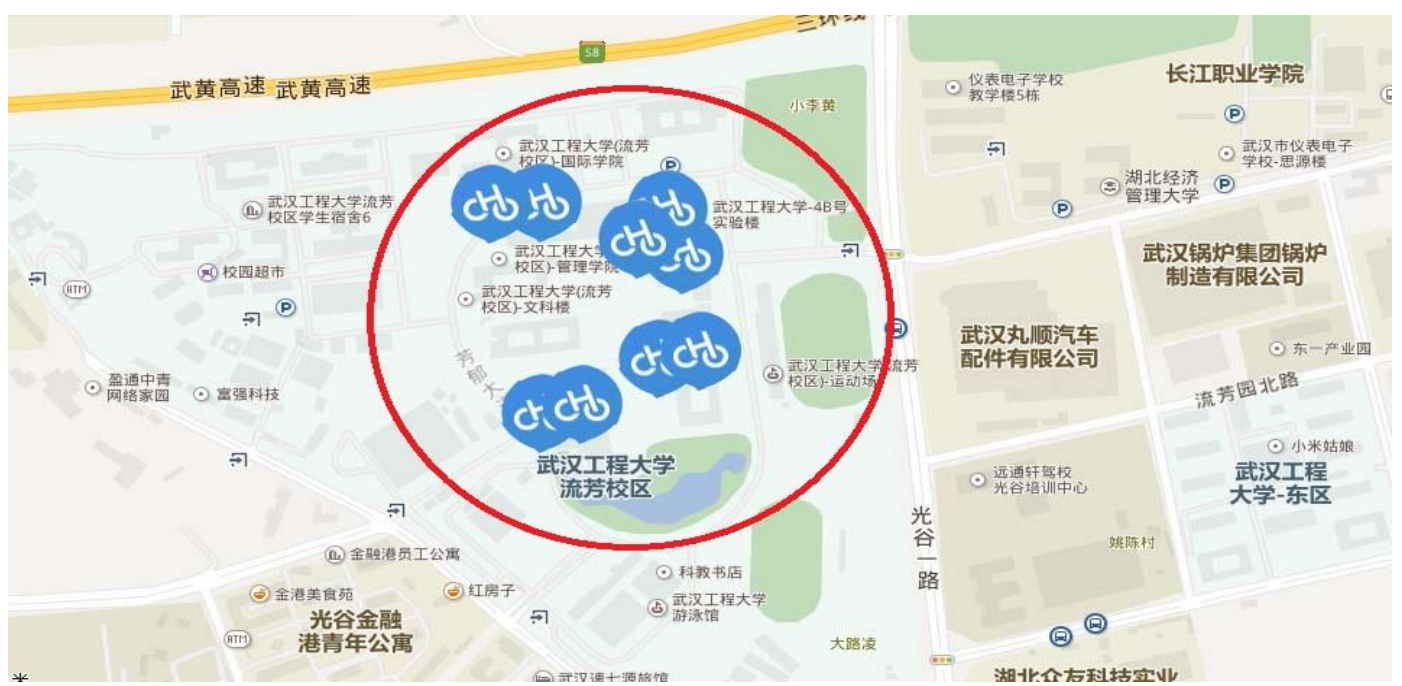

Figure 4 . The effect of the vehicle on the map immediately after entering the circle area.

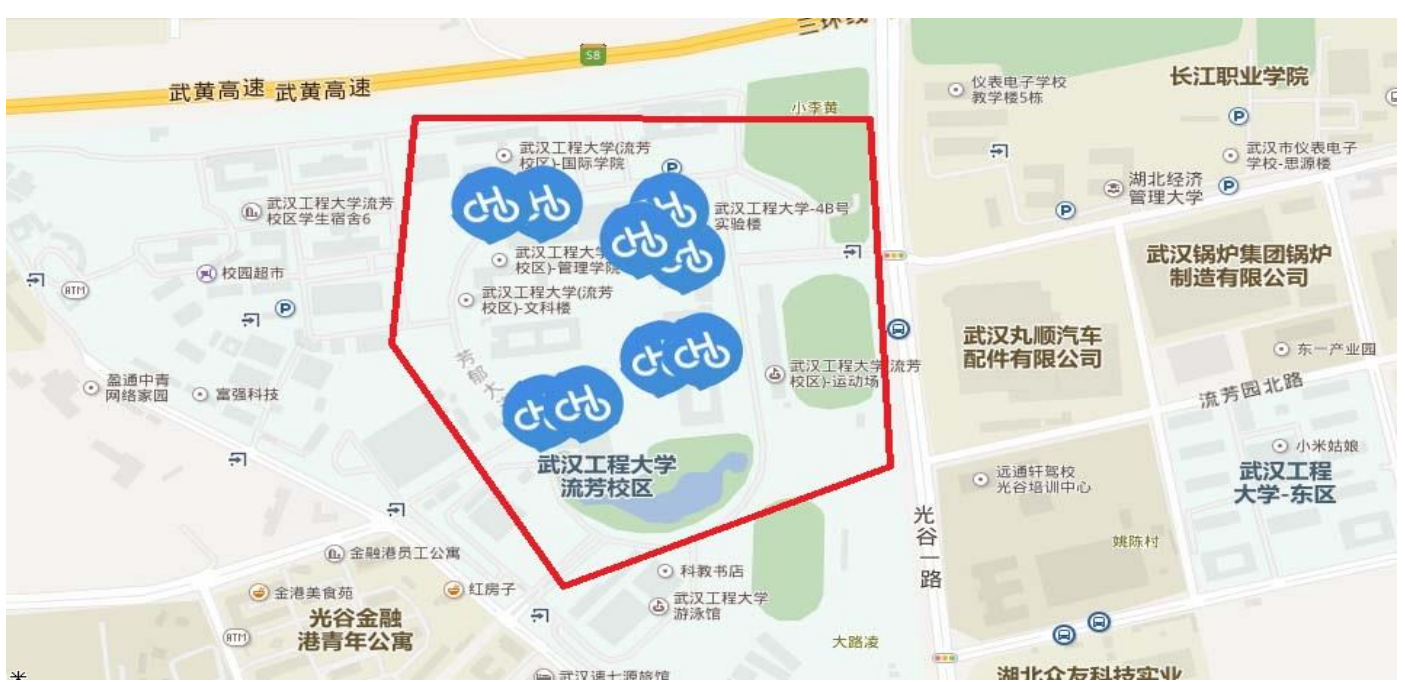

Figure 5. The effect of the vehicle on the map immediately after entering the polygon area.

\section{References}

[1] Zhou xiping, jiang bin. Design and implementation of electronic fence in vehicle monitoring [J].Computer Knowledge and Technology, 2016, 12(13):107-109.

[2] Sun Shibo. GPS vehicle positioning system based on GPRS and its evidence inference map matching algorithm [D]. Harbin Institute of Technology, 2006(6).

[3] Li Cong. Design and implementation of map matching algorithm [D]. Beijing Jiaotong University, 2011(6).

[4] GPS,http://zh.wikipedia.org/wiki/GPS.

[5] Gao Zhengdong, Yan Jiefeng, Huang Qiang. Realization of electronic fence in GPS vehicle monitoring system [J]. Computer Knowledge and Technology, 2009, 5(6), p1380-1381. 